\title{
Railspeak: outil de communication orale spécifique pour le TGV transmanche
}

\section{Pascale Fade}

\section{CpenEdition}

\section{Journals}

Édition électronique

URL : http://journals.openedition.org/asp/4393

DOI : $10.4000 /$ asp. 4393

ISSN : 2108-6354

Éditeur

Groupe d'étude et de recherche en anglais de spécialité

Édition imprimée

Date de publication : 1 mars 1993

Pagination : 449-461

ISSN : 1246-8185

Référence électronique

Pascale Fade, «Railspeak: outil de communication orale spécifique pour le TGV transmanche », ASp [En ligne], 1 | 1993, mis en ligne le 03 juin 2014, consulté le 03 mai 2019. URL : http:// journals.openedition.org/asp/4393; DOI : 10.4000/asp.4393

Ce document a été généré automatiquement le 3 mai 2019.

Tous droits réservés 


\title{
Railspeak: outil de communication orale spécifique pour le TGV transmanche
}

\author{
Pascale Fade
}

1 Dans le cadre de la mise en place de liaisons ferroviaires TGV entre Paris \& Londres et Londres \& Bruxelles en 1993, la SNCF et ses partenaires British Rail et SNCB doivent tenir compte de certains aspects spécifiques à ces nouvelles lignes. Celles-ci présentent en effet les caractéristiques suivantes: utilisation d'une technologie encore récente, le Train à grande vitesse, dont le TGV Transmanche sera la dernière évolution; interpénétration de réseaux ferroviaires ; passage dans le Tunnel sous la Manche.

2 Pour la SNCF, initiateur et leader du projet, il s'agit donc, en collaboration avec les autres compagnies, de répondre à des problèmes d'une part ferroviaires et, d'autre part, linguistiques.

3 Les problèmes ferroviaires, qui ne relèvent pas de notre compétence mais que nous intégrons néanmoins dans notre réflexion, sont les suivants : création du nouveau TGV qui doit desservir ces lignes et, en particulier, ergonomie de la cabine du conducteur; formation des agents de conduite à ce nouveau type de train ; apprentissage de la ligne par les agents, à la fois sur le sol français et sur le sol britannique; ceci pose en particulier le problème de la connaissance du système ferroviaire britannique et de ses spécificités ; inconnue que représente actuellement le Tunnel.

4 Les problèmes linguistiques, que nous sommes amenés à traiter avec la SNCF, seront l'objet de cet exposé. Après un bref historique de la situation, nous définirons le concept de communication orale formalisée retenu dans le cadre de ces liaisons Transmanche, et nous envisagerons les principes linguistiques qui doivent sous-tendre l'élaboration de cette communication. Il est indispensable de préciser que la phase d'élaboration ellemême n'a pas encore commencé et que cet exposé correspond à un rapport d'étape qui représente à la fois l'aboutissement d'une réflexion et le point de départ du travail de création de cette communication orale formalisée. 


\section{Historique}

5 Dans les liaisons ferroviaires internationales actuelles, le passage d'une frontière implique quasi systématiquement le changement de l'agent de conduite; ceci en particulier pour des raisons de sécurité : méconnaissance du système étranger, de son fonctionnement, de ses procédures... Dans le cas du TGV Transmanche, cette condition n'a pas été retenue pour diverses raisons : d'une part la rapidité de la liaison n'imposait pas un changement de conducteur en cours de trajet, d'autre part la SNCF, promoteur et chef de file du projet, n'a pas souhaité ce changement pour des raisons techniques et politiques. Outre les problèmes ferroviaires cités précédemment, liés à l'interpénétration de réseaux, se posait alors la question de la communication entre les conducteurs de TGV français et les personnels au sol britanniques, et inversement. Pour traiter cette question, la SNCF s'est adressée au Groupe Interacadémique Langues (G.I.L.) qui a confié le dossier à l'Académie de Nancy-Metz, dossier pris en charge par Cité Langues ${ }^{1}$, département de la Délégation académique à la Formation continue (DAFCO). L'étude qui a débuté en 1989 est menée sur la base d'un partenariat entre la SNCF et Cité Langues. Compte tenu, entre autres, des enjeux et des objectifs assignés, elle implique également une collaboration étroite avec European Passenger Services (EPS, société créée par British Rail dans le cadre spécifique des liaisons Transmanche) et leur partenaire linguistique, Polytechnic of Central London (PCL). La SNCB et Eurotunnel sont également associés à cette étude.

6 La demande formulée initialement par la SNCF était à la fois simple et imprécise - ou pourrait-on dire, précise et complexe, comme bon nombre de demandes linguistiques émanant d'entreprises: " Vu que nos agents de conduite du futur TGV Transmanche seront amenés à communiquer oralement en anglais, nous souhaiterions leur proposer une formation adaptée tant au contexte d'utilisation qu'aux exigences de sécurité ».

7 Une première étude de faisabilité a été menée afin, d'une part, de mieux cerner le contexte ferroviaire ainsi que la nature des exigences et, d'autre part, de déterminer les compétences langagières (nature et degré) requises. Cette première étude a abouti à la conclusion suivante: un degré élevé de maîtrise de la langue est nécessaire afin de garantir que la communication entre les conducteurs de TGV français et les personnels au sol britanniques s'effectue avec succès et réponde aux exigences de sécurité. La SNCF a rejeté cette solution pour deux raisons essentielles:

8 - il est matériellement impossible de consacrer beaucoup de temps à la formation linguistique des conducteurs étant donné les délais relativement courts de mise en service du TGV Transmanche et étant donné la priorité à accorder à la formation professionnelle ferroviaire de ces personnels ;

9 - il n'est pas souhaitable, d'un point de vue psychologique, que l'exigence linguistique soit trop forte et vienne concurrencer l'exigence de qualification ferroviaire. Ceci est en relation avec la gestion des ressources humaines au sein de la SNCF.

10 Compte tenu de ce cahier des charges, la réflexion s'est portée sur la recherche d'une solution qui soit à la fois réaliste en terme de compétences à acquérir et garante d'une efficacité optimale pour répondre aux enjeux de sécurité. 


\section{Exigences linguistiques de l'interpénétration de réseaux ferroviaires}

\subsection{Contexte ferroviaire}

11 Dans des conditions habituelles, les moyens techniques ferroviaires mis en place sur les lignes TGV (balises au sol qui déclenchent un signal en cabine) et sur les lignes normales (signaux visuels et sonores) assurent le bon déroulement et la sécurité d'un trajet. Toutefois des conditions inhabituelles peuvent survenir et compromettre alors ce bon déroulement (rails endommagés, non-fonctionnement d'un signal). C'est dans ces circonstances particulières, et uniquement celles-ci, qu'une communication orale entre les conducteurs de TGV Transmanche et les personnels au sol devra s'établir, afin de répondre aux exigences immédiates et absolues. La communication orale sera donc exceptionnelle, justifiée par l'urgence de la prise en compte d'un événement ou par des conditions extraordinaires nécessitant une information sur-le-champ.

Le cadre dans lequel s'effectuera cette communication peut être défini comme suit :

13 - seuls les personnels au sol et les conducteurs de TGV utiliseront ce mode de communication ;

14 - le recours à la communication orale se fera : train en mouvement, train à l'arrêt.

15 - dans la situation "train en mouvement ", la communication orale portera uniquement sur l'arrêt du train. En effet, le conducteur est concentré sur sa conduite et la lecture de ses différents instruments de bord et, pour des raisons de sécurité, il n'est pas envisageable de le détourner de ses préoccupations premières.

16 - dans la situation " train à l'arrêt », la communication orale portera sur les circonstances qui ont justifié cet arrêt et sur les conditions de redémarrage du train ou sur le maintien à l'arrêt.

17 - la langue utilisée pour la communication sera le français ou l'anglais selon le pays dans lequel se trouve le train. En ce qui concerne le Tunnel, Eurotunnel envisage la présence de deux agents, l'un français, l'autre anglais, à chaque poste de travail.

18 Étant donné les enjeux, la sécurité en particulier, cette communication orale devra être la plus efficace et la plus rentable possible, viser l'essentiel et éviter le superflu, tout en offrant un degré optimal d'intelligibilité. En outre, il est inconcevable, également pour des raisons de sécurité, qu'une consigne formulée à l'oral ne puisse pas être comprise ni appliquée par le destinataire sans délai.

19 De plus, en terme de compétences requises, il serait utopique de vouloir recruter ou former des conducteurs français, anglais et belges à un degré de bilinguisme total qui pourrait permettre de communiquer oralement en langue étrangère sans gêne et sans risque d'erreur.

20 Compte tenu de ce contexte, la réponse adéquate, et seule capable de garantir le respect des exigences et des contraintes, réside dans le concept de communication orale formalisée. 


\subsection{Concept de communication orale formalisée}

21 Cette communication orale formalisée sera construite à partir d'un ensemble de situations de communication répertoriées avec l'aide des partenaires ferroviaires et elle sera constituée d'un ensemble fini (limité en nombre) de dialogues formalisés faisant intervenir deux personnes au maximum. L'initiateur de ces dialogues étant soit le conducteur, soit le personnel au sol. Cet ensemble de dialogues formalisés devra être autosuffisant, c'est-à-dire devra couvrir tous les besoins de communication et prévoir toutes les circonstances inhabituelles évoquées précédemment. Le concept de formalisation signifie que tous les dialogues seront prédéterminés, élaborés à l'avance, c'est-à-dire que le contenu même sera prédéfini. En d'autres termes, il s'agit de construire des dialogues en nombre nécessaire et suffisant qui englobent les différentes données, à la fois situationnelles et communicatives.

Ces dialogues en nombre fini, connus par tous les utilisateurs, sont les garants d'une bonne communication, c'est-à-dire l'émission et la réception d'informations mutuellement comprises et qui vont droit au but. En effet, l'emploi de dialogues formalisés garantit la précision, la non-ambiguïté et la concision (dire «bref » et " clair »). Ils évitent les détails encombrants et inutiles, et limitent la communication au minimum indispensable. Par exemple, dans la situation où des animaux encombreraient les voies, un message d'information du type "animaux sur la voie » correspondrait au minimum indispensable alors que des renseignements concernant le nombre précis, la race de ces animaux, seraient des détails encombrants qui allongeraient inutilement le message et pourraient retarder l'accomplissement d'une action urgente.

Les dialogues formalisés sont une garantie mutuelle de bonne compréhension et de réaction rapide de la part des utilisateurs car ne sont entendus que des messages connus et qui seront donc reconnus très rapidement. Dans le domaine de la sécurité, il est essentiel que toute action soit prise "à propos » et qu'aucune initiative hasardeuse ne survienne. Le redémarrage d'un train à l'arrêt est une action qui suit l'ordre de redémarrer car l'ordre a été compris, et non une initiative prise suite à une compréhension incertaine d'un message.

Les dialogues formalisés doivent permettre enfin de répondre de façon fiable à une caractéristique essentielle de cette communication, sa rareté d'utilisation. Il est indispensable de prendre en compte que le recours à la communication orale sera l'exception, motivé par des circonstances particulières. Ce qui signifie que la communication orale pourrait éventuellement ne jamais intervenir mais que les participants potentiels doivent toujours être en mesure de l'utiliser. En d'autres termes, il s'agit de s'assurer que les compétences en langue acquises lors d'une formation seront bien présentes et disponibles le moment venu, quel que soit le délai entre cette formation et le moment de l'utilisation. La réponse à ce décalage entre la formation et la mise en pratique est bien l'utilisation d'une communication orale formalisée rapidement mise en place : le moment venu, il n'est pas question d'hésiter ou d'improviser, la compétence doit être totale. Le recours à des dialogues prédéterminés, fixés à l'avance, est le seul garant de cette compétence. L'utilisation différée des compétences acquises impose que ces compétences puissent être efficacement entretenues et par conséquent bien délimitées. aucun cas rentrer dans ce cadre; les "figures imposées» sont la réponse. La 
communication orale formalisée a donc été adoptée par les différents partenaires en réponse à ce besoin de communication orale spécifique. Toutefois, le concept même étant défini, il s'imposait de déterminer les règles qui devaient gouverner cette communication.

Nous avons alors établi une liste de principes linguistiques généraux que chaque dialogue, anglais ou français, devra respecter lors de son élaboration afin de garantir une homogénéité parfaite entre les différents dialogues.

\section{Principes linguistiques généraux}

27 Je ne reprendrai ici que certains de ces principes, les plus significatifs.

\subsection{Composition des dialogues formalisés}

Compte tenu des procédures de transmission (radio, téléphone) et de «ce qui doit faire l'objet de la communication entre les personnels au sol et les conducteurs de TGV", chaque dialogue formalisé sera constitué des deux types de messages suivants :

Les messages de procédures VHF formalisés, qui doivent obéir aux règles dictées par la spécificité du canal de communication: ils concernent exclusivement le processus d'établissement, de maintien et de clôture du contact radio ou téléphone. Exemple: identification de l'émetteur (ici Lille), du récepteur (train 2.3.1.8), clôture de la communication (terminé)...

Les messages ferroviaires formalisés qui correspondent aux échanges (informations, instructions, avertissements...) dictés par les conditions ferroviaires du moment.

De plus, ces deux types de messages formalisés comporteront des variables situationnelles qui concernent le numéro du train, le nom de la station sol, la position et le déplacement sur la voie, les références dans le livret de procédures, la vitesse, le temps et l'heure.

32 En cas de mauvaise compréhension de tout ou partie d'un message formalisé, des contrôleurs de compréhension seront utilisés. Ils serviront soit à indiquer à l'autre une erreur de compréhension, soit à vérifier sa propre compréhension auprès de son interlocuteur.

33 En d'autres termes, les dialogues comporteront deux types de messages prédéterminés, les messages VHF et les messages ferroviaires et, dans ces messages, l'utilisateur incorporera à l'endroit réservé la ou les variables appropriées à sa situation. Par exemple dans le message suivant: "Marche à vue jusqu'au PK 2.8 ", le message ferroviaire prédéterminé est "Marche à vue jusqu'au PK ", la variable appropriée à la situation et choisie par l'utilisateur est 2 et 8.

34 Par ailleurs, à ces deux types de messages obligatoires qui constituent le dialogue pourront à tout moment s'ajouter des contrôleurs de compréhension, tels qu'ils viennent d'être définis. C'est-à-dire que l'élaboration des dialogues va effectivement prévoir un déroulement standard de chaque dialogue tout en mettant à la disposition des utilisateurs des « outils de dépannage » pour remédier à un problème de compréhension.

Nous n'avons malheureusement pas le temps ici de lister les contrôleurs de compréhension ni d'évoquer leur réalisation et leur emploi. 


\subsection{Les identificateurs de message}

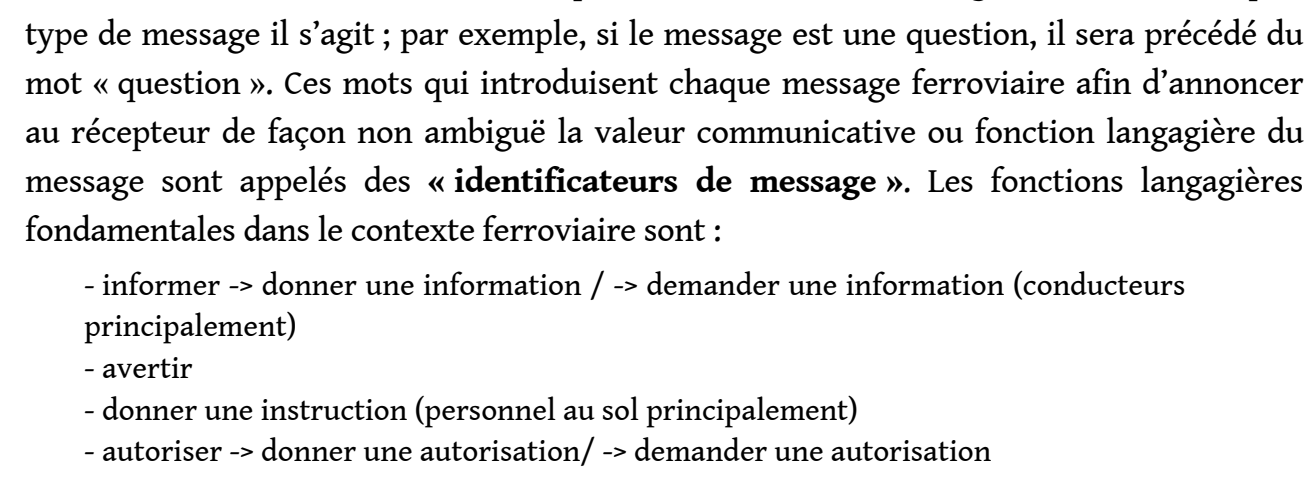
type de message il s'agit ; par exemple, si le message est une question, il sera précédé du mot «question». Ces mots qui introduisent chaque message ferroviaire afin d'annoncer au récepteur de façon non ambiguë la valeur communicative ou fonction langagière $d u$ message sont appelés des «identificateurs de message ». Les fonctions langagières fondamentales dans le contexte ferroviaire sont :

Les identificateurs de message qui correspondent à ces fonctions langagières seront: information, question, avertissement, instruction, autorisation, demande d'autorisation.

\subsection{Confirmation de réception}

Tout message ferroviaire devra faire l'objet d'une confirmation de réception : il n'est pas suffisant de transmettre une information, il faut également s'assurer que l'information est correctement reçue. Pour s'assurer de l'accord mutuel sur ce qui a été transmis, il est nécessaire d'une part d'utiliser un identificateur de réception, et d'autre part de répéter le message dans son intégralité. L'identificateur de réception sera: « reçu ».

Exemple : Instruction : Redémarrer le train.

Reçu. Instruction : Redémarrer le train.

(Identificateur de message ferroviaire : Instruction.

Message ferroviaire formalisé : Redémarrer le train.

Identificateur de réception : Reçu.

Répétition du message dans son intégralité)

En quelques mots, les autres principes linguistiques concernent le caractère univoque de chaque message, le nombre de messages par transmission, les règles concernant les variables situationnelles, etc.

En plus de ces principes linguistiques généraux, il était nécessaire d'envisager les principes linguistiques d'élaboration des dialogues eux-mêmes.

\section{Principes linguistiques d'élaboration des dialogues formalisés}

41 étant donné les enjeux liés à l'efficacité de la communication orale, en particulier la sécurité, la concision, la brièveté et la non-ambiguïté des messages et des dialogues formalisés sont les critères fondamentaux retenus pour leur élaboration.

Dans le domaine lexical, les principaux critères sont les suivants :

- éviter ce qui est difficile à prononcer pour un étranger,

- éviter les faux-amis, les mots ambigus, les mots trop longs,

- éviter le jargon ferroviaire très spécifique à une langue, 
- favoriser les correspondances entre langues (préférer, par exemple, « animaux » à « bétail », « animaux » étant proche de « animals » en anglais).

Dans le domaine syntaxique,

- éviter les formulations trop longues,

- éviter les raccourcis qui peuvent générer des incompréhensions,

- définir des structures simples et standard pour chaque type de message ; ce qui signifie que les questions seront formulées d'une manière, les informations d'une autre...

En ce qui concerne l'intonation, elle ne doit jouer aucun rôle dans le sens des messages : toute valeur communicative habituellement véhiculée par l'intonation doit être signifiée par d'autres « éléments » linguistiques ou communicatifs.

Il est utile de repréciser que la phase d'élaboration des dialogues eux-mêmes n'est pas encore commencée, et que nous n'avons donc pas encore testé systématiquement l'applicabilité de l'ensemble de ces principes. Un accord entre les différents partenaires sur l'ensemble des points qui viennent d'être évoqués est en cours, et cet accord permettra de passer à la phase d'élaboration. Il appartiendra alors à chaque pays de créer l'ensemble des dialogues formalisés en respectant tous les principes énoncés: nos partenaires britanniques élaboreront les dialogues anglais, nous, Français, élaborerons les dialogues français. Il est prévu, ensuite, que chaque équipe valide le travail de l'autre.

efois, certaines questions restent en suspens, et celle-ci en particulier : est-il possible d'envisager que les choix lexicaux et syntaxiques varient d'une langue à l'autre selon la culture ferroviaire du pays et selon l'usage? prévisibilité et des attentes du récepteur, il est souhaitable que la réalisation d'un message dans sa langue d'origine privilégie les règles et conventions qui lui sont connues. Ceci est vrai à la fois pour l'anglais et le français. Si les règles sont similaires dans les deux langues, il n'y a pas de problème. Si les règles sont différentes, faut-il respecter systématiquement les règles et conventions de la langue utilisée ou peut-on introduire des modifications linguistiquement acceptables et qui faciliteraient la communication de l'utilisateur étranger? Il n'est pas question d'envisager « a mickey-mouse language » ou de s'exprimer en "petit nègre" mais des choix sont à faire en tenant compte des conséquences qu'ils impliquent. Il est certainement envisageable de favoriser les correspondances entre langues, mais il est nécessaire de délimiter les « cas à problème " afin d'en connaître l'ampleur et d'y apporter une solution.

Parallèlement à l'outil de communication, un point essentiel est la formation des conducteurs de TGV.

\section{Application des concepts et principes dans la formation}

47 La maîtrise de la communication orale formalisée spécifique ferroviaire pose deux problèmes majeurs.

Comment concevoir la formation pour qu'elle débouche sur une compétence opérationnelle des conducteurs? En d'autres termes, quel contenu définir, quelles techniques d'apprentissage mettre en œuvre..., pour que l'évaluation finale soit à $100 \%$ positive, atteste d'une maîtrise sans faille? 


\section{BIBLIOGRAPHIE}

Johnson, E. 1990. « Policespeak: Frontier research for a new frontier». In Actes du XI colloque du GERAS, Bordeaux.

Robertson, F.A. 1988. Airspeak: Radiotelephony communication for pilots. Londres : Prentice Hall.

Weeks, F. et al. 1984. Seaspeak Reference Manual. Oxford : Pergamon Press.

Weeks, F. et al. 1988. Seaspeak Training Manual. Oxford : Pergamon Press.

\section{NOTES}

1. Cité Langues (Conseil, Ingénierie, Technologie, Études en Langues, 28 rue de Saurupt 54000 Nancy.

\section{AUTEUR}

\section{PASCALE FADE}

Université Nancy 2. pascale.fade@univ-lorraine.fr 